\title{
What do they mean? Using Media Richness as an Indicator for the Information Value of Stock Analyst Opinion regarding post-earnings Firm Performance
}

\author{
Matthias Eickhoff \\ University of Göttingen \\ Matthias.Eickhoff@wiwi.uni-goettingen.de
}

\begin{abstract}
In this research the impact of media-richness on the investor reaction to earnings announcements is investigated. To this end, unstructured (high-richness) sources of analyst opinion are subjected to text-mining and combined with structured (low-richness) sources of analyst opinion, as well as other commonly used structured data relevant to company performance. Results indicate that equivocality is a major problem faced by investors, while uncertainty as understood by media-richness theory appears to be less dominant.
\end{abstract}

\section{Introduction}

The quarterly earnings announcements of publically traded companies pose significant challenges for both academia and investors alike. Research regarding this quarterly ritual is extensive and often concerns the impact of the newly revealed information on the expectations of investors. Meanwhile, investors face a more ad-hoc decision problem. They need to decide whether to buy, to hold, or to sell a share on the ground of so far announced information. In todays networked society, many information sources are available to investors. Among those, the variant opinions of stock analysts are a key source of analysis regarding past and future company performance. However, these opinions are available over a multitude of channels including both financial information systems, which provide structured recommendations, telephone earnings calls, which are publically available in most jurisdictions, and analyst reports, which interested investors can purchase. This poses a selection problem for investors: Which of these sources are (is) most important regarding the investment decision? This research addresses that decision problem using media richness theory as a background on which different media types can be evaluated regarding a number of criteria. Furthermore, media richness theory provides pointers on what kind of media type (rich or less rich) is more efficient, given the presence of uncertainty and equivocality in any given decision problem. On this basis, the available media types are evaluated and tested regarding their explanatory power as to historical investment decisions, which are measured as the abnormal stock return following earnings announcements. Section 2 provides a brief introduction to recent finance and accounting research concerning earnings releases and the related sources of analyst opinion Following, it discusses the relation of media richness theory to the investment decision problem. Section 3 introduces the data used in the following analysis. Section 4 gives a methodological overview and shows how sentiment analysis, topic-mining and the estimation of abnormal returns are performed as a basis for this research. Consequently, section 5 shows how the analysis is performed and presents its results. Section 6 discusses the implication of the presented results before this research is concluded in section 7 .

\section{Theory}

\subsection{Analyst opinion}

Earnings releases in general and earnings call transcripts in particular have been studied regarding their information content by continuous streams of accounting and finance research. Previous work focusses on structured information regarding the earnings release itself and non-textual measures regarding the earnings call. The information content of earnings announcements have been found to be stable over several decades regarding both abnormal trading volume and return volatility [1-3]. More recently, research focusses on textual measures derived from the transcripts of earnings' calls. Commonly, studies use wordlists based measures to capture call tone [4]. Also, a lack of spontaneity in management responses to analyst questions in earnings calls has been investigated [4]. Furthermore, investment reaction to analyst tone is usually stronger than to management tone [1]. In line with these findings, positive tone increases the effect of positive earnings surprise [5]. Moreover earnings forecasts published by analysts who participated in earnings' calls immediately after 
them are more accurate than those published by analysts who did not participate [6]. It has been shown that allowing analysts to participate in conference calls gives them access to private information, even though the calls are public, and management can use such measures to discriminate against unfavorable analysts in earnings calls [7]. Still, earnings calls have been found to be a valuable source of information for investors. Likewise, the reports and non-earnings' calls related forecasts released by stock analysts have been subject to continuous interest by accounting and finance researchers. As is the case in the area of earnings' announcements, earlier research focused on structured recommendations released by analysts. One source for these structured analyst opinions is given by the institutional broker estimate system $(\mathrm{I} / \mathrm{B} / \mathrm{E} / \mathrm{S})$, in which analysts publish and continuously update estimates on numerous financial indicators related to a company's performance. On the basis of such data, research has shown that mean forecasts (the average opinion of all analysts submitting an estimate) overemphasizes the common information all analysts share over the private information that makes the estimates interesting in the first place [8]. In addition analysts with historically more accurate forecasts are more likely to make bold predictions (as opposed to sticking to the consensus estimate) than those with poorer forecast accuracy in their past [9].

Like the purely earnings related analyst opinion research, this research stream has also begun to analyze textual analyst opinions, which are presented in analyst reports and examined the choice of peer companies used by sell-side equity analysts [10]. On the same note it's showing that report readability correlates with analyst capability [11]. It has also been shown that report tone can provide excess information beyond structured forecasts [12].

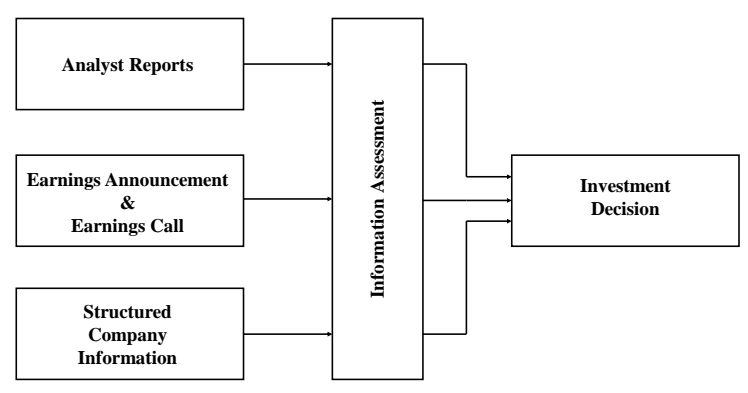

Figure 1: Investment Decision

As this overview of research on analyst opinion shows, research regarding the textual sources of analyst opinion has begun, but so far has mainly focused on the augmentation of traditional models regarding the accuracy and impact of analyst opinion. As shown by this prior research, this textual content can improve upon the structured recommendations given by analysts through $\mathrm{I} / \mathrm{B} / \mathrm{E} / \mathrm{S}$ and similar systems. This research contributes towards this growing corpus of knowledge by investigating two of the reasons why this is so using established IS theory. Furthermore, topic-mining is used to extract information about the impact of specific topics discussed in both analyst reports and earnings calls. In this study, the investor reaction to the release of quarterly earnings will be considered in conjunction with the release of analyst reports and estimates for earnings surprise. Figure 1 shows this basis for investment decisions following earnings announcements.

\subsection{Media Richness Theory:}

Media Richness Theory as proposed by Daft and Lengel analyzes the effectiveness of different media types regarding the transportation of information between different individuals or organizations [13]. It argues that in order to convey information effectively the transport medium needs to match the complexity of the transmitted information regarding four core criteria: (1) Language variety, (2) multiplicity of cues (channel variety), (3) personalization (source), and (4) feedback immediacy. Language variety does not necessarily refer to the use of different natural languages but the mediums' capability to transmit a wide spectrum of concepts and ideas. For example, Daft and Lengel list music and art as media with a high language variety in their seminal work on the subject, as opposed to mathematics as an example of a low variety language. Cue multiplicity alludes to the variety in channels through which a medium transmits information. In example, face-to-face communication offers more channels (facial expressions, audio, visual) than a phone call (audio). Personalization or the source of communication refers to the soft factor of being able to interact with another person instead of a machine or written communication. Finally, feedback immediacy extends this notion by allowing to correct faulty perceptions by the recipient of transmitted information. If a medium ranks high across these categories, it is considered rich. Based upon this richness categorization the theory argues that rich media types perform superior to less rich media types in equivocal tasks, while less rich media types can support information transmission in the presence of uncertainty [14]. Uncertainty categorizes situations in which a decision maker has not been supplied with enough information to reach a well based decision. Equivocality describes a situation in which the decision maker is faced with numerous and possibly 
conflicting sources of information, making it difficult to reach a firm decision [15, 16]. Earnings announcements present both uncertain and equivocal problems to decision makers (investors). On the one hand, the investor wants to assess the future performance of a company based on information, which in the best case presents the current state of the company. As all predictions are, this assessment is highly uncertain. On the other hand, the investor is faced with numerous opinions pertaining to the subject at once. The management of a company will often interpret a given situation differently than stock analysts, media, or the investor. Consequently, the information presented to the investor is highly equivocal. Assuming both of these assumptions hold true, media richness theory suggests that there is good reason to listen to both low and high richness media types regarding earnings announcements. Low richness media types may help to reduce uncertainty, while high richness media types may mitigate equivocality. This leads to the question if the hypothesized effects are measurable in media types related to earnings announcements. To address this question, the following research Questions are proposed:

RQ1: Do low richness media types transmit investment-relevant information regarding earnings announcements, i.e. does low media complexity help to investors to reduce uncertainty after earnings announcements?

RQ2: Do high richness media types transmit investment-relevant information regarding earnings announcements, i.e. do increased language variety, cue multiplicity, personalization and feedback immediacy in their union increase the information content of materials related to earnings announcement by reducing equivocality? These isolated considerations naturally lead to a third question considering the combination of both high and low richness media types:

RQ3: What is the incremental value of high and low richness media types when their antipode is already being considered?

\section{Structured, unstructured Data and their Relation to Media Richness Theory}

In this section the data types used in the following analysis are presented. In the context of media richness theory, structured and unstructured data can be considered as extreme representations of low and high richness media types. Typically, structured data is highly formalized and consequently scores low in the discussed categories, assessing media richness. In contrast, unstructured data typically consists of higher richness media types, such as earnings call transcripts and analyst reports in our case. Thus, sources of structured and unstructured data can serve as proxies for low and high richness media types. All data used in this study is obtained from Thompson Reuters' Datastream and Advanced Analytics (TRAA) platforms.

\subsection{Low Richness (Structured Data)}

Three kinds of low richness data sources of interest to investors are investigated in this study. First, the stock price of companies in the sample is collected. Secondly, several balance sheet related variables, commonly used in relation to earnings announcements are added to augment the stock price. Finally, several analyst consensus estimates are collected from $\mathrm{I} / \mathrm{B} / \mathrm{E} / \mathrm{S}$, which reflect the mean estimates of all analysts who had submitted their opinion about each variable on the call date. Table 1 provides a detailed description of each collected variable. Prior research regarding the effects of analyst opinion on investor behavior shows that investors do listen to this lowrichness information source. For example, trading strategies based on the consensus estimate have been analyzed [17], as have the effects of boldness on forecast accuracy [18].

Table 1: Variable Description

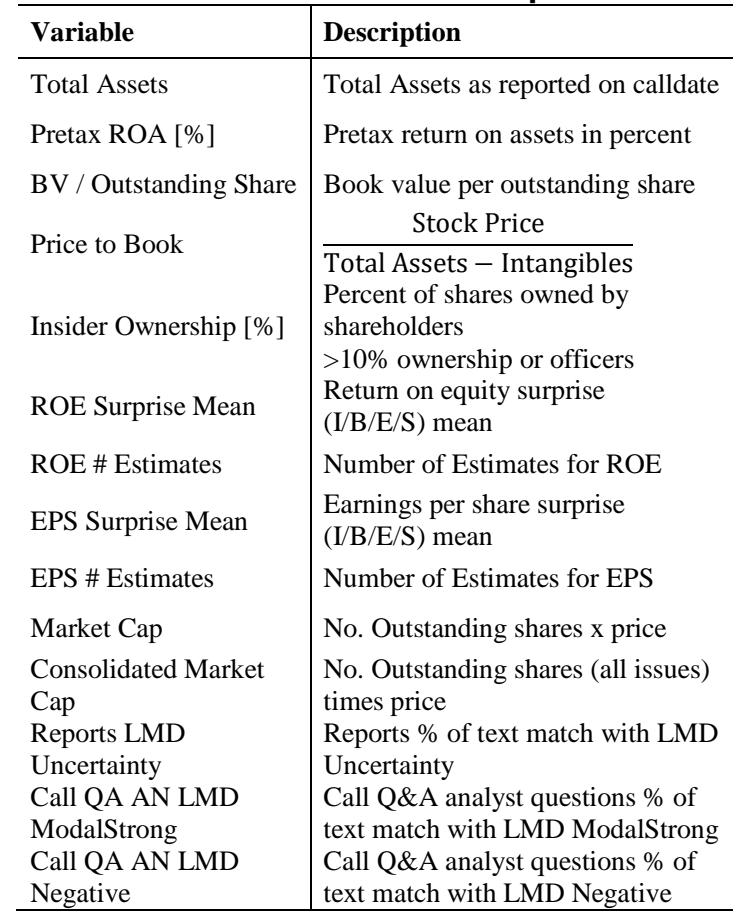



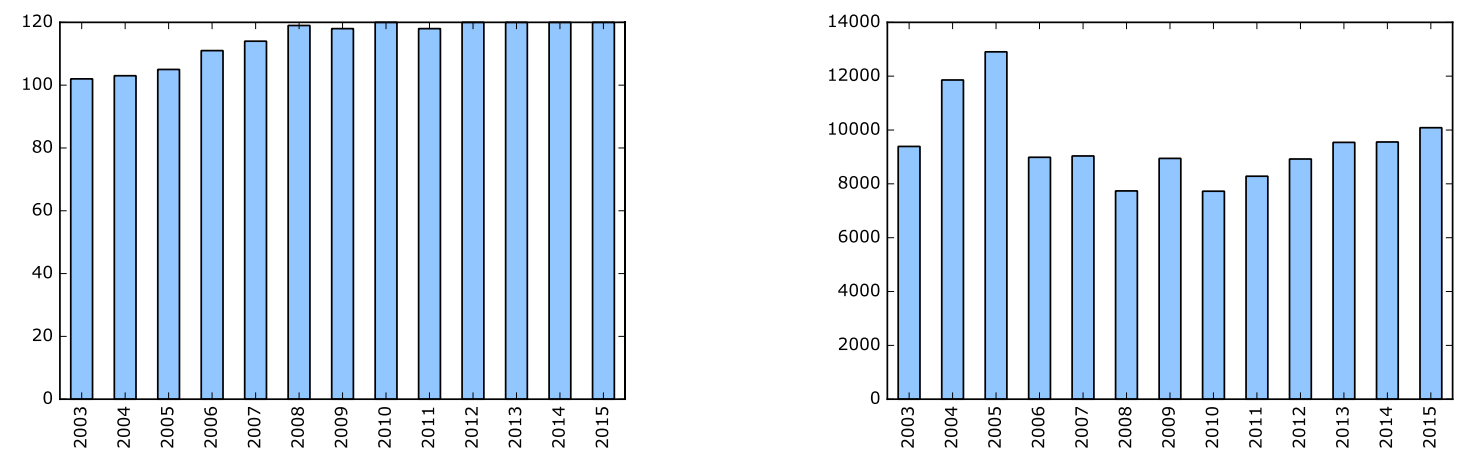

Figure 2: Histograms of call (left) and report (right) counts.

\subsection{High Richness (Unstructured Data)}

Two sources of unstructured analyst opinion data are used in the following analysis. Both were collected from Thompson Reuters Advanced Analytics (TRAA). Both analyst reports and earnings conference calls have been studied regarding to their effect on investor behavior. Analyst reports have been studied regarding the market reaction to their release [19], the effect of their readability on abnormal trading volumes [11], and effect of report ambiguity on investor reaction [20]. Likewise, investors' reactions to earnings conference calls have been studied in regard to the link between effects of call tone and investor sophistication [5], as well as the effects of call tone on abnormal returns [21]. As these prior studies have repeatedly shown, these sources of unstructured but high-richness media often lead to significant investor reactions. Thus, both low- and high-richness media sources have been shown to be feasible predictors for investor behavior. The following data are used in this analysis. At first, analyst reports about the companies included in the Dow Jones Industrial Average $\left(\right.$ DJIA $\left._{30}\right)$ between 2003 and 2016 were collected. These analyst reports typically contain a review of the current financial situation of the company and an estimate of its future development, in a mixture between freely written text as well as tables and figures.

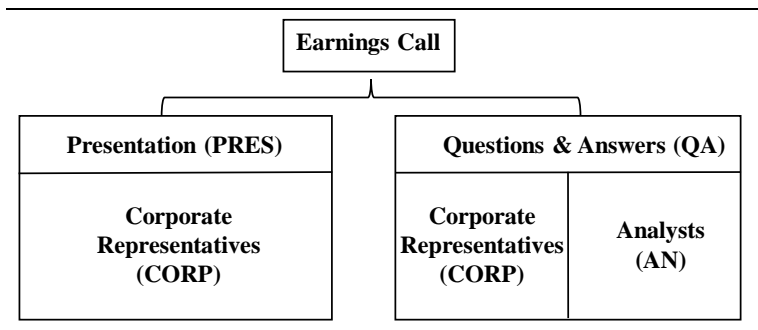

Figure 3: Earnings Call Structure.
This paper focuses on analyzing the freely written portion of the reports and consequently extracting the textual content of each document for further analysis. Second, the transcripts of all earnings calls for the same period were collected. Earnings calls typically consist of two separate segments. First, the management of the company holding the call presents the earnings announcement in form of a monologue of the $\mathrm{CEO}$ or CFO. Second, analysts ask questions about the announcement or other topics of interest for possible future company performance. Figure 3 gives an overview of this structure. Three types of contribution to the call, i.e. presentation by the corporation, questions by analysts, and answers by the corporation, are extracted to calculate separate measures for each. If a call does not exhibit all three segments it was dropped from the sample. Figure 2 shows the annual counts for each media type. In the case of the earnings calls 120 per year is the natural limit for a 30 company $\left(\mathrm{DJIA}_{30}\right)$ sample (one call per quarterly earnings per year). As shown, data are available continuously for the analyzed period with a notable reduction in the report count during the global financial crisis ( 2008).

\section{Method}

In this section, an overview of the methodologies and tools utilized in this paper will be provided. First, the text mining methods used to extract variables from textual content are elaborated. Second, the abnormal return model is developed. Finally, the commonly used methods to analyze the resulting data are presented.

\subsection{Sentiment Analysis}

Sentiment Analysis involves extracting the emotional contents of documents or document collections with the intension of providing an 
overview on the opinions and feelings of their authors. Both supervised and unsupervised learning has been applied to this field of content analysis [22]. While both approaches have been used with success, clearly certain trade-offs exist using either. On the one hand, supervised learning can offer excellent accuracy but domain-portability poses a challenge [23]. On the other hand, dictionary based (unsupervised) sentiment scoring is inherently limited by the dictionary of sentiment laden words texts are compared to, resulting in the need for domain-appropriate sentiment dictionaries [24]. As this analysis intends to asses both analyst reports and earnings call transcripts and finance specific sentiment dictionaries are available, the dictionary based approach is chosen. Two dictionaries to score both types of texts were chosen, a general purpose dictionary developed by $\mathrm{Hu}$ and Liu with Positive and Negative categories [25] and a finance specific dictionary developed by Loughran and McDonald (LMD) with Positive, Negative, Litigious, Modal-Weak, Modal-Strong and Uncertainty categories [26]. In the case of analyst reports, a 30-day report sample prior to each earnings call event is averaged regarding these categories. For the calls themselves, the three discussed segments are scored individually for each call.

\subsection{Topic Mining}

In contrast to sentiment analysis, topic mining aims to extract what is being said in a document and not how authors feel about a specific topic. Latent Dirichlet Allocation (Table 2) [27, 28] is a topic mining algorithm and generates a pre-determined number of topics in a document collection D consisting of a vector of documents $\mathbf{w}$, each of which consists of $\mathbf{N}$ individual words $\mathrm{w}_{\mathrm{n}}$. A topic $\mathrm{Z}_{\mathrm{n}}$ is represented as a mixture of the words contained in the document collection. In turn, each topic is assigned to each document with a certain probability. For the purpose of our analysis the topic-assignments to each earnings call in the sample and the average of the topic assignments to all analyst reports 30 days prior to the call date (equivalent to the sentiment measures) are computed using the MALLET topic mining package [28]. For the calls themselves, topics are computed for the entire call and not for the three individual segments. The main reason behind this is, that the Q\&A portion of the call often contains very short text parts, i.e. short questions which are asked by analysts often receive short answers, and consequently are not as suited for being analyzed by using LDA on each individual text. The topic model is trained on all textual data at once, i.e. both analyst reports and the conference call transcripts are used as training data.
Table 2: Latent Dirichlet Allocation.

1. Choose $\mathbf{N} \sim \operatorname{Poisson}(\xi)$

2. Choose $\Theta \sim \operatorname{Dirichlet}(\alpha)$

3. For each of the $\mathbf{N}$ words $w_{n}$ :

I: $\quad$ Choose topic $\mathrm{z}_{\mathrm{n}} \sim \operatorname{Multinomial}(\Theta)$

II: Choose a word $w_{n}$ from $p\left(w_{n} \mid z_{n}, \beta\right)$, i.e. the multinomial conditional probability of the word conditioned on the topic $\mathrm{z}_{\mathrm{n}}$.

The model is set to identify 100 topics. The number of topics is the crucial model parameter in our case. If the parameter is chosen too low, the resulting topics lack granularity if it is chosen too high the resulting topics are increasingly indistinguishable for humans. As noted, a topic consists of the likelihood that each word in the corpus is part of the topic. Consequently, topics can be very similar in regard to their most likely words but very different in their overall composition, consequently making them hard to distinguish. The number of 100 topics is determined by experiments keeping this trade-off in mind and seems reasonable as this relatively high number of topics helps to avoid company specific topics, i.e. topics that simply classify a text regarding the company it belongs to. To validate the number of chosen topics, a HDP (hierarchical dirichlet process) model [29] was trained using Gensim [30]. This model, depending on the cutoff-likelihood (relative importance of one topic compared to others), points to 80-95 topics as the 'optimal' amount. As the following analysis uses a variable selection approach, choosing a slightly higher number seems reasonable as superfluous topics will not be included in the resulting models. Another option is to train the topic model dependent on companies or industries with lower individual topic numbers. The main reason this was not done here is that it would result in topics that do not allow for intercompany or inter-industry comparisons of topic impacts.

\subsection{Abnormal Returns}

The analysis of the investment decisions following an earnings announcement aims to capture the reaction of the average investor to said announcement. It is important to keep in mind that only changes in opinion can be measured on the stock market. In general, three possible reactions to an earnings announcement are conceivable. First, an investor can be positively surprised by the announcement and consequently buy the share. Second, the opposite reaction follows a 
Table 3: Topics relevant in regression models.

\begin{tabular}{l|l|l|r}
\hline Type & ID & Label (Coding) & Top Words \\
\hline $\mathrm{R}$ & 8 & Earnings & report securities andor affiliates financial eps companies subject \\
$\mathrm{R}$ & 43 & Credit Cards & volume growth debit payments payment credit card revenue \\
$\mathrm{R}$ & 51 & Agreement & agreement announced technology company systems development health \\
$\mathrm{R}$ & 63 & Chem. Products & sales materials company chemicals performance protection products segment \\
$\mathrm{R}$ & 69 & Investment & report information investment price research securities limited financial \\
$\mathrm{R}$ & 72 & Pharma Products & cancer disease phase products infections trial life science \\
$\mathrm{R} / \mathrm{C}$ & 75 & Pharma Research & sales patients data phase product products drug study \\
$\mathrm{R} / \mathrm{C}$ & 86 & Rating & research report securities investment companies stock industry months \\
$\mathrm{R}$ & 94 & Sports & footwear apparel brand growth china futures product athletic \\
$\mathrm{C}$ & 35 & Listing & listed listing sales investext data deleted services corporate \\
$\mathrm{C}$ & 37 & Risk & rating report markets firm risk global securities investment \\
$\mathrm{C}$ & 66 & Prudence & prudential group equity llc rating york report analyst \\
$\mathrm{C}$ & 88 & Financial Data & source exhibit data research yoy survey figure index \\
$\mathrm{C}$ & 99 & Markets & world markets report company sector securities investment research \\
\hline
\end{tabular}

negative surprise. However, if an investor does not change her opinion and consequently does not alter her position regarding a stock, no trade occurs and consequently no change in price can be observed on the market. Nonetheless, both negative and positive changes in opinion should lead to a change in the investors' position and consequently would be observable on the market. Thus, the measurement is strictly limited to the unexpected portion of the information contained in an earnings announcement. In order to monitor the aggregate change in investor opinions, after an earnings announcement, the abnormal return of a company's share after the event can serve as a proxy. This approach to measuring investor opinion relies on the efficient market hypothesis, which stipulates that any new information should be represented in the stock price immediately $[31,32]$. In order to monitor the abnormal component of returns following an event, an expectation of the normal return for the same period in the absence of the event needs to be formulated and the abnormal return is defined as the difference between observed and expected returns following the event: $A R_{i, t}=R_{i, t}-$ $E\left(R_{i, t} \mid X_{t}\right) . E\left(R_{i, t} \mid X_{t}\right)$ refers to the expected return given $X_{t}$, the development of a reference group (S\&P500) of shares during the period. The estimation of these normal returns is performed by using the market model approach [33], assuming a timeconstant relation between the reference group and the stock in question, using the following OLS model: $R_{i, t}=\alpha_{i}+\beta_{i} R_{M}+\epsilon_{i, t} \quad$ with $E\left(\epsilon_{i, t}\right)=0 \quad$ and $\operatorname{Var}\left(\epsilon_{i, t}\right)=\sigma_{\epsilon, i}^{2}$ using -200 days up to -1 day prior to the earnings announcement as training data for the return model. This yields the abnormal return for a given day, starting with the day of the earnings announcement itself. Indeed, both $\mathrm{AR}_{\mathrm{t} 0}$ to $\mathrm{AR}_{\mathrm{t} 10}$ and cumulative return measures (CARs) were calculated for the sample. $\mathrm{AR}_{0}$, the abnormal returns on the day of the earnings call, was most suitable for the analysis and consequently is used as the independent variable for the resulting models.

\subsection{Topic Selection}

As noted, the topic model trained on earnings call transcripts and analyst reports is used to compute the topic composition for each call transcript in the sample and the average topic composition of all analyst reports released 30 days prior to the call. As each of these two topic groups consists of 100 topics, this alone results in numerous topic-variables per call event. As it is infeasible to use all 200 topic-variables in the following regression models and more importantly it is unknown which topics computed by the model, are of interest to investors, a selection needs to be performed. A two-step approach to this selection problem is chosen. First, a topic set suitable for regression is identified using the least absolute shrinkage and selection operator (LASSO) [34], which selects a subset of variables out of the pool of topics. The sentiment based variables are also supplied to the LASSO. The selected sentiment variables are those included in the regression models in Table 4. Second, because automated variable selection by itself is problematic, the resulting topic set is manually inspected as a sanity check. The coding is performed by looking at the top 20 words for each topic (10 displayed for space reasons). The resulting topic set is displayed in Table 3. As shown, a label is assigned to each topic by coding the top words of each selected topic. The type column indicates whether the topic was selected to be relevant in calls (C), analyst reports (R), or both. This has two purposes: Firstly, only topics, which are interpretable, should be used for further analysis. Secondly, it helps to make the following regression models easier to read, by replacing the topic numbers with the resulting codes. As shown, a mixture of topics indicating discussions of both financial topics and company or industry specific topics was selected. This points to the possibility of estimating the topic 
Table 4: Regression model $\left(Y=A R_{0}\right)$ summaries.

\begin{tabular}{|c|c|c|c|c|c|c|}
\hline & \multicolumn{2}{|c|}{ M1: Structured Data } & \multicolumn{2}{|c|}{ M2: Unstructured Data } & \multicolumn{2}{|c|}{ M3: Both (M1 + M2) } \\
\hline & Coefficient & SE & Coefficient & SE & Coefficient & SE \\
\hline Total Assets & -0.027 & 0.104 & & & -0.093 & 0.091 \\
\hline Pretax ROA [\%] & -0.101 & 0.105 & & & 0.012 & 0.099 \\
\hline BV / Outstanding Share & 0.046 & 0.187 & & & 0.048 & 0.19 \\
\hline Price to Book & $0.511 * * *$ & 0.152 & & & $0.461 * * *$ & 0.157 \\
\hline Insider Ownership [\%] & 0.065 & 0.074 & & & 0.022 & 0.065 \\
\hline ROE Surprise Mean & $-0.430 * *$ & 0.178 & & & $-0.393 * *$ & 0.163 \\
\hline ROE \# Estimates & 0.009 & 0.117 & & & 0.072 & 0.109 \\
\hline EPS Surprise Mean & -0.106 & 0.168 & & & -0.177 & 0.179 \\
\hline EPS \# Estimates & 0.013 & 0.088 & & & -0.085 & 0.082 \\
\hline Consolidated Market Cap & $-1.945^{*}$ & 0.994 & & & $-2.573 * *$ & 0.999 \\
\hline Market Cap & $1.898^{*}$ & 0.998 & & & $2.602 * *$ & 1.006 \\
\hline Reports Topic Earnings & & & $0.111^{*}$ & 0.061 & 0.1 & 0.065 \\
\hline Reports Topic Credit Cards & & & $-0.131 * *$ & 0.061 & $-0.141 * *$ & 0.061 \\
\hline Reports Topic Agreement & & & $-0.148 * *$ & 0.061 & $-0.141 * *$ & 0.063 \\
\hline Reports Topic Chem. Products & & & $-0.133 * *$ & 0.06 & $-0.148 * *$ & 0.066 \\
\hline Reports Topic Investment & & & $0.140 * *$ & 0.06 & $0.137 * *$ & 0.062 \\
\hline Reports Topic Pharma Products & & & -0.069 & 0.061 & 0.007 & 0.085 \\
\hline Reports Topic Pharma Research & & & $-0.125 * *$ & 0.061 & $-0.115^{*}$ & 0.062 \\
\hline Reports Topic Rating & & & 0.082 & 0.193 & 0.221 & 0.197 \\
\hline Reports Topic Sports & & & $0.157 * *$ & 0.062 & $0.197 * * *$ & 0.065 \\
\hline Call Topic Listing & & & 0.077 & 0.064 & 0.112 & 0.073 \\
\hline Call Topic Risk & & & 0.068 & 0.079 & 0.05 & 0.079 \\
\hline Call Topic Prudence & & & $-0.188 * * *$ & 0.062 & $-0.190 * * *$ & 0.063 \\
\hline Call Topic Pharma Research & & & 0.123 & 0.08 & $0.136^{*}$ & 0.078 \\
\hline Call Topic Rating & & & 0.074 & 0.194 & -0.242 & 0.216 \\
\hline Call Topic Financial Data & & & $0.119 * *$ & 0.06 & $0.133 * *$ & 0.062 \\
\hline Call Topic Markets & & & $0.129 * *$ & 0.059 & $0.126^{* *} *$ & 0.06 \\
\hline Reports LMD Uncertainty & & & $0.102 *$ & 0.061 & $0.166^{* *} *$ & 0.067 \\
\hline Call QA AN LMD ModalStrong & & & $0.126 * *$ & 0.061 & $0.126^{* * *}$ & 0.061 \\
\hline Call QA AN LMD Negative & & & $-0.198 * * *$ & 0.067 & $-0.188 * * *$ & 0.068 \\
\hline Constant & 0 & -0.068 & 0 & 0.058 & 0 & 0.056 \\
\hline Observations & 206 & & 206 & & 206 & \\
\hline R2 & 0.089 & & 0.381 & & 0.443 & \\
\hline Adjusted R2 & 0.038 & & 0.318 & & 0.348 & \\
\hline Residual Std. Error & 0.981 & $\mathrm{df}=194$ & 0.826 & $\mathrm{df}=186$ & 0.808 & $\mathrm{df}=175$ \\
\hline F Statistic & $1.730 *$ & $\mathrm{df}=11 ; 194$ & $6.030 * * *$ & $\mathrm{df}=19 ; 186$ & $4.642 * * *$ & $\mathrm{df}=30 ; 175$ \\
\hline
\end{tabular}

models on industry specific samples to generate more granularity. However, this would require a dataset covering a larger index than the DJIA because within this index only a couple of companies per industry are represented.

\section{Analysis and Results}

The topics selected using the described approach are combined with the variables obtained from structured data sources and for all variables z-scores are computed $(z=(x-\mu) / \sigma)$ in order to normalize the scale of the variables. It is important to keep this step in mind when interpreting the following regression models since the coefficients refer to the deviation from the mean of each variable, i.e. a negative coefficient refers to a value smaller than the average value of the variable and vice-versa. All calls on which the full set of variables could not be obtained were dropped from the sample. This mainly concerns two types of missing variables. First, calls for which no more than 5 reports were available 30 days prior to the earnings release were dropped in order to obtain better averages. Second, the $\mathrm{I} / \mathrm{B} / \mathrm{E} / \mathrm{S}$ estimates were not available for earlier years. In principle, some calls could be reintegrated after variable selection is performed but the smaller sample size is kept as this seemed like the cleaner approach. In order to analyze the data with regard to the research questions three models need to be estimated. The first model (M1) only contains variables from the structured data sources and addresses RQ1 (Do low richness media types transmit investment relevant information?). The second model (M2) only contains variables estimated from unstructured data and addresses RQ2 (Do high richness media types transmit investment relevant information?). The third model (M3) contains both types of variables addresses RQ3 (What incremental value can be gained by combining both low and high richness media types?). Regarding the interpretation of these models and their connection to the research questions three aspects are of particular interest. Firstly, the coefficient sizes within the individual models and their stability (especially regarding their sign) across the models. Secondly, which coefficients are significant across the different models? And thirdly, and most interestingly, how to the models 
compare to one another on the model level. Table 4 shows the three resulting models.

Regarding M1 media richness theory predicts that low-richness media types should help information transmission by mitigating issues of uncertainty (RQ1). As M1 contains such low-richness information, it should be able to explain the investment decisions following earnings announcements if uncertainty is a relevant problem for this decision. As shown, the adjusted $\mathrm{R}^{2}$ of this model is comparatively small, indicating that the variables obtained from structured data are not able to explain much of the variance within the abnormal returns on the call day. Keeping this in mind, both price to book ratio and unconsolidated market capitalization show significant positive effects, while return on equity surprise mean and consolidated market capitalization exhibit negative effects. Overall, while the examined structured data offers some insight regarding the investment decisions following the earnings call, the comparatively small adjusted $\mathrm{R}^{2}(3.8 \%)$ points at a small effect of uncertainty as described by media richness theory for this decision type.

Regarding M2 media richness theory predicts that high-richness media types should help information transmission by mitigating issues of equivocality (RQ2). Thus, M2 should be able to explain the investment decisions following earnings announcements if equivocality is a relevant problem for decision makers. As shown, the adjusted $\mathrm{R}^{2}$ $(31.8 \%)$ of this model is comparatively large, indicating that the variables extracted from unstructured content explain a larger portion of the variance within abnormal stock returns on the earnings call day. Thus, these unstructured data sources seem to transmit more investment relevant information when compared to the structured data sources contained in M1. Within the framework of media richness theory this points to equivocality regarding the interpretation of earnings announcements as a major problem investors need to mitigate using high-richness media sources. Within the topics of analyst reports released in a period of 30 days before the earnings calls several topics are identified that allude toward a positive effect of the earnings announcement. In particular, the discussion of earnings, investments and sports (likely and industry specific topic) show positive coefficients. Likewise, the discussion of financial data and market activity in the call itself shows positive effects. Finally, strong modal words from the LMD word list used by analysts in their Q\&A questions relate to positive postcall returns.

On the other hand, reports containing credit cards, corporate agreements, chemical products, pharma research and prudence exhibit negative effects. Of these, especially the prudence topic is interesting because it isn't as industry specific as the other negative topics. Finally, negative words used by analysts in their Q\&A questions show a negative relation to returns. Overall, this model explains a much larger portion of return variance than the one incorporating traditional structured data, thus indicating that equivocality seems to be a bigger problem than uncertainty regarding the investment decisions after earnings calls. Finally, M3 combines both low- and high-richness media types in one model and investigates the complementary value of this combination beyond the value of the individual models (RQ3). As shown, this model slightly improves upon the adjusted $\mathrm{R}^{2}$ from $31.8 \%$ to $34.8 \%$, which means the improvement is slightly smaller than the adjusted $\mathrm{R}^{2}$ of M1. This finding is in line with the intention of the measure, which penalizes models including more covariates. With regard to RQ3, this relatively small improvement over M2 may be interpreted as a small incremental value of combining low- and highrichness media types. However, keeping the limitations of the chosen text mining approaches in mind, which do not extract all information contained in the unstructured data, it is doubtful, if this incremental value would be present if an informed investor actually reads the analyst reports and listens to the earnings call. Both of the above also may contain the relevant information contained in the structured data sources. Thus, the incremental value of the combined model can be doubted. Still, it can serve as a stability check for M2. As shown, the addition of the variables contained in M1 does not alter the sign of the significant variables of M2. The only change in covariate significance is given by the pharma research topic, which is not significant in M2 but is in M3.

In summary, the models indicate a low impact of uncertainty for post-earnings call investment decisions, while providing much clearer evidence for the impact of equivocality. Finally, the incremental value of the combination of low- and high-richness media types is doubtful if the low impact of uncertainty as understood by media richness theory and the information likely contained in the unstructured content but not captured by text-mining approaches are kept in mind.

\section{Implications and Limitations}

The implications of the presented results can be spilt up into practical, theoretical and policy implications. From a practical perspective, investors should focus both on analyst reports and earnings calls as their primary sources of information regarding the impact of earnings announcements, if no automation is required or text mining is feasible. Still, when this is not the case the available structured data can support investment decisions. Even when these reports explained much less of the variance within abnormal 
returns in this study. Also, as also shown by other studies [1, 2, 5, 21, 35], the presented analysis reinforces the crucial effect of call and report tone towards investor reaction. The theoretical contribution of this study is twofold. First, its results contribute towards the growing body of research regarding the impact of unstructured, high-richness, media types in accounting and finance research by exploring the feasibility of topic-mining within the problem domain of earnings announcements. Secondly, it contributes towards the discussion of media richness theory and its applications by exploring the effects of uncertainty and equivocality in regard to the same problem domain. On a whole results indicate that equivocality presents a major challenge to investors. Finally, results indicate that policy makers should keep a watchful eye on the private information gained by analysts because of their privileged access to top level corporate representatives as the high correlation to abnormal post-earnings returns may point to the presence of such private information (or analysts simply to a good job). The presented results should be interpreted only while keeping the following limitations in mind. As shown, some of the topics that are identified by the chosen approach are industry specific. Thus, an extension of the chosen approach to a larger sample with industry specific topics is desirable. Also, the identified topics are time-constant and a topic model can only identify topics, which are previously contained in a corpus. Thus, entirely new topics will be missed and would be interesting to add in the future. Furthermore, the observation count in the final regression models is limited by data availability regarding structured analyst consensus estimates. Like the inclusion of industry specific topics, this could possibly be mitigated, by using a larger index as the basis of the analysis (data collection would pose a significant hurdle to such an extension).

\subsection{Future research}

The discussed limitations and presented results outline several avenues for future research. First, future research can examine the industry specific effects of analyst opinion on investor reaction to earnings announcement and their relation to low and rich media types. Secondly, many other media types are of interest to investors. In example, social media has been used to explain stock returns [36]. Also, the audio versions of earnings calls present an opportunity to work with higher-richness media than with their transcripts. Furthermore, as noted in the limitations, the presented topic model does treat all calls and report equally over time. However, of course the topics discussed in earnings calls and analyst reports change over time. For example, during the global economic crisis call topics were likely much more negative than the model based on all data can represent and viceversa. This point of topic sentiment hints at another possible extension. The per-topic sentiment of analyst opinion represents a final possible addition to the presented approach. Finally, other topic modelling techniques, dynamic topic models [37], exist and a comparison of their suitability for post earnings call return analysis provides an opportunity for future research.

\section{Conclusion}

This study examined the impact of earnings-call related low- (structured) and high-richness (unstructured) content on abnormal stock returns on the earnings-call day before the background of media richness theory. Results indicate that uncertainty poses a smaller challenge to investors than equivocality, i.e. that the decision problem of investors is dominated not by a lack of clarity or information availability but a lack of consensus among the available information sources. Furthermore, the complementary value of combining low- and high-richness media types is examined but results regarding this combination are unclear. While combined models show minor improvements over one only including high-richness media, it is doubtful if this improvement would be present if investors manually examine high-richness media sources. Additionally, results reinforce the evidence for the relation between analyst tone and investor reaction to analyst opinion and explore topicmining as another information extraction technique that can aid in understanding the content of analyst communication.

\section{References}

[1] P. Brockman, X. Li, and S. M. Price, "Differences in Conference Call Tones: Managers vs. Analysts," Financial Analysts Journal, vol. 71, pp. 24-42, 2015.

[2] A. K. Davis, W. Ge, D. Matsumoto, and J. L. Zhang, "The effect of manager-specific optimism on the tone of earnings conference calls," Review of Accounting Studies, vol. 20, pp. 639-673, 2015.

[3] W. R. Landsman and E. L. Maydew, "Has the information content of quarterly earnings announcements declined in the past three decades?," Journal of Accounting Research, vol. 40, pp. 797-808, 2002.

[4] J. Lee, "Can Investors Detect Managers' Lack of Spontaneity? Adherence to Predetermined Scripts during Earnings Conference Calls," The Accounting Review, vol. 91, pp. 229-250, 2015.

[5] B. M. Blau, J. R. DeLisle, and S. M. Price, "Do sophisticated investors interpret earnings conference call tone differently than investors at large? Evidence from short sales," Journal of Corporate Finance, vol. 31, pp. 203-219, 2015. 
[6] W. J. Mayew, N. Y. Sharp, and M.

Venkatachalam, "Using earnings conference calls to identify analysts with superior private information," Review of Accounting Studies, vol. 18, pp. 386-413, 2013.

[7] W. J. Mayew, "Evidence of management discrimination among analysts during earnings conference calls," Journal of Accounting Research, vol. 46, pp. 627-659, 2008.

[8] O. Kim, S. C. Lim, and K. W. Shaw, "The inefficiency of the mean analyst forecast as a summary forecast of earnings," Journal of Accounting Research, vol. 39, pp. 329-335, 2001.

[9] O.-K. Hope, "Accounting policy disclosures and analysts' forecasts," Contemporary Accounting Research, vol. 20, pp. 295-321, 2003.

[10] G. De Franco, O.-K. Hope, and S. Larocque, "Analysts choice of peer companies," Review of Accounting Studies, vol. 20, pp. 82-109, 2015.

[11] G. Franco, O.-K. Hope, D. Vyas, and Y. Zhou, "Analyst report readability," Contemporary Accounting Research, vol. 32, pp. 76-104, 2015.

[12] A. H. Huang, A. Y. Zang, and R. Zheng, "Evidence on the information content of text in analyst reports," The Accounting Review, vol. 89, pp. 2151-2180, 2014.

[13] R. L. Daft and R. H. Lengel, "Information richness. A new approach to managerial behavior and organization design," 1983.

[14] R. L. Daft and R. H. Lengel, "Organizational information requirements, media richness and structural design," Management science, vol. 32, pp. 554-571, 1986.

[15] R. L. Daft and N. B. Macintosh, "A tentative exploration into the amount and equivocality of information processing in organizational work units," Administrative science quarterly, pp. 207224, 1981.

[16] A. R. Dennis and S. T. Kinney, "Testing media richness theory in the new media: The effects of cues, feedback, and task equivocality,"

Information Systems Research, vol. 9, pp. 256274, Sep 1998.

[17] B. Barber, R. Lehavy, M. McNichols, and B. Trueman, "Can investors profit from the prophets? Security analyst recommendations and stock returns," The Journal of Finance, vol. 56, pp. 531-563, 2001.

[18] M. B. Clement and S. Y. Tse, "Financial analyst characteristics and herding behavior in forecasting," The Journal of Finance, vol. 60, pp. 307-341, 2005.

[19] P. Asquith, M. B. Mikhail, and A. S. Au, "Information content of equity analyst reports," Journal of financial economics, vol. 75, pp. 245282, 2005.

[20] J. Winchel, "Investor Reaction to the Ambiguity and Mix of Positive and Negative Argumentation in Favorable Analyst Reports," Contemporary Accounting Research, vol. 32, pp. 973-999, 2015.

[21] S. M. Price, J. S. Doran, D. R. Peterson, and B. A. Bliss, "Earnings conference calls and stock returns: The incremental informativeness of textual tone," Journal of Banking \& Finance, vol. 36, pp. 992-1011, 2012.

[22] B. Liu, "Sentiment analysis and opinion mining," Synthesis Lectures on Human Language Technologies, vol. 5, pp. 1-167, 2012.

[23] A. Aue and M. Gamon, "Customizing Sentiment Classifiers to New Domains : a Case Study," Proceedings of Recent Advances in Natural Language Processing RANLP, vol. 49, pp. 207$18,2005$.

[24] A. Muhammad, N. Wiratunga, R. Lothian, and R. Glassey, "Domain-Based Lexicon Enhancement for Sentiment Analysis," in SMA@ BCS-SGAI, 2013, pp. 7-18.

[25] M. Hu and B. Liu, "Mining and summarizing customer reviews," Proceedings of the 2004 ACM SIGKDD international conference on Knowledge discovery and data mining KDD 04, vol. 04, pp. 168-168, 2004.

[26] T. Loughran and B. McDonald, "When is a liability not a liability? Textual analysis, dictionaries, and 10-Ks," The Journal of Finance, vol. 66, pp. 35-65, 2011.

[27] D. M. Blei, A. Y. Ng, and M. I. Jordan, "Latent dirichlet allocation," The Journal of Machine Learning Research, vol. 3, pp. 993-1022, 2003.

[28] A. K. McCallum, "MALLET: A Machine Learning for Language Toolkit," ed, 2002.

[29] Y. W. Teh, M. I. Jordan, M. J. Beal, and D. M. Blei, "Hierarchical Dirichlet Processes," Journal of the American Statistical Association, vol. 101, pp. 1566-1581, 2006.

[30] R. Řehůřek and P. Sojka, "Software Framework for Topic Modelling with Large Corpora," in LREC 2010 Workshop on New Challenges for NLP Frameworks, Valletta, Malta, 2010, pp. 4550.

[31] E. F. Fama, L. Fisher, M. C. Jensen, and R. Roll, "The adjustment of stock prices to new information," International economic review, vol. 10, pp. 1-21, 1969.

[32] B. G. Malkiel and E. F. Fama, "Efficient capital markets: A review of theory and empirical work," The Journal of Finance, vol. 25, pp. 383-417, 1970.

[33] A. C. MacKinlay, "Event studies in economics and finance," Journal of economic literature, vol. 35, pp. 13-39, 1997.

[34] R. Tibshirani, "Regression shrinkage and selection via the lasso," Journal of the Royal Statistical Society. Series B (Methodological), pp. 267-288, 1996

[35] B. Twedt and L. Rees, "Reading between the lines: An empirical examination of qualitative attributes of financial analysts' reports," Journal of Accounting and Public Policy, vol. 31, pp. 121, 2012.

[36] J. Bollen, H. Mao, and X. Zeng, "Twitter mood predicts the stock market," Journal of Computational Science, vol. 2, pp. 1-8, 2011.

[37] D. M. B. J. D. Lafferty, "Dynamic_Topic_Models," in 23'rd International Conference on Machine Learning, Pittsburgh PA, 2006, pp. 113--120. 